\title{
Bibliotecas digitales para niños y adolescentes: psicología del desarrollo y diseño de sistemas de información
}

Digital libraries for children and young adults: developmental psychology and information systems design

\section{Francisco Javier GARCíA MARCo}

Facultad de Filosofía y Letras, Universidad de Zaragoza, 50009 Zaragoza, jgarcia@unizar.es.

\begin{abstract}
Resumen
Se analiza el desarrollo psicosical de las primeras etapas de la vida para mostrar como los factores psicológicos y psicosociales deben ser tenidos en cuenta en el desarrollo de sistemas de información y bibliotecas digitales para niños y adolescentes. Se destacan tres aspectos del diseño de bibliotecas digitales y servicios de información para niños y adolescentes que deben tenerse especialmente en cuenta: el contenido, las características perceptuales y simbólicas de la interfaz y las herramientas de comunicación. Se proporcionan pistas concretas para el desarrollo eficaz en cada área. Se concluye que la individuación y la socialización como tendencias claves del desarrollo psicosocial se correlacionan con dos de las grandes tendencias en el desarrollo de sistemas de información -la personalización y la interacción social-y que, por ello, deben estudiarse las interacciones entre ambos desarrollos, pues forman un campo privilegiado para la investigación interdisciplinar.
\end{abstract}

Palabras clave: Bibliotecas digitales. Niños. Adolescentes. Aspectos psicológicos. Aspectos sociales. Computación social. Personalización.

\section{Introducción: personalización y socialización}

En este artículo se pretende reflexionar sobre una de las dimensiones de variabilidad más importantes en los sistemas de información y, particularmente, en las bibliotecas digitales: la propia evolución del sujeto humano a lo largo de su vida, la evolución psicosocial, que determina tanto las capacidades con las que los sujetos pueden acometer los procesos de búsqueda de información como sus necesidades e intereses, $\mathrm{y}$, por tanto, de forma definitiva, el éxito de un sistema de información. Se aborda específicamente el periodo de la niñez y la adolescencia, que es cuando el sujeto humano experimenta la mayor parte de su desarrollo físico, cognitivo y social, que se puede resumir en dos procesos de cambio complementarios y dialécticos -en

\begin{abstract}
The psychosocial development in the first stages of life is analyzed to show how the psychological and psychosocial factors must be taken into account in the design of information systems, and, particularly, of digital libraries for children and teenagers. It is concluded that three aspects are of critical importance: content, the perceptual and symbolic characteristics of the interface, and the communication tools. Specific tips in each area are provided. Finally, it is concluded that a correlation exists among the main trends in psychosocial development -individuation and socialization - and two of the main tendencies in information systems design - personalization and social interaction-; so, it can be affirmed that they form together a privileged field for interdisciplinary research.
\end{abstract}

Keywords: Children digital libraries. Juvenile digital libraries. Social aspects. Psychological factors. Social computing. Librarian role. Personalization.

el sentido de interactivos y no exentos de conflicto-: la individuación y la socialización.

Dicho en términos del diseño moderno de sistemas de información, la personalización y interacción social son dos de las tendencias más importantes en el diseño de sistemas de información y bibliotecas digitales en la actualidad, pero, sobre todo, son dos de las tendencias básicas en la evolución de los sujetos humanos a lo largo de su vida. En este trabajo, se pretende explorar esas raíces y obtener implicaciones teóricas y prácticas para el diseño de sistemas de información.

\subsection{Personalización}

Un aspecto fundamental del diseño de interfaces humanos es la denominada "personaliza- 
ción", esto es, la adaptación del sistema a la idiosincrasia de los individuos de manera que éstos puedan interaccionar mejor con él, y lograr que el sistema mayor que incorpora al usuario y al sistema funcione en su conjunto de forma más eficaz y eficiente.

Un caso extremadamente sencillo de personalización es la capacidad de los reproductores de audio para que el usuario pueda ajustar el volumen según su capacidad de audición, el sonido ambiente, etc.

La personalización ofrece ventajas tanto desde el punto de vista del usuario como del proveedor de servicios -entendiendo también que todo producto supone un servicio-. El usuario puede ajustar el comportamiento del sistema a sus condiciones personales y ambientales. El fabricante puede atender a más población objetiva, minimizando la insatisfacción que se produce en aquellos clientes cuyas capacidades y necesidades se alejan de la media en aspectos identificados y predecibles.

Es más, la personalización ha sido postulada como una de las claves del desarrollo económico en las sociedades de libre mercado, en las cuales se pasa de la producción masiva de objetos iguales para un consumidor tipo a una oferta de productos cada vez más personalizados, más de nicho, orientados a consumidores específicos, implicados en el diseño y desarrollo de los productos y exigentes, a los que Alvin Toffler (1980) denominó prosumidores.

En la práctica, la personalización procede mediante la "segmentación" de las características del sistema en función de una serie de propiedades en las que la población de usuarios objetivo varía.

De esta forma, el diseñador parametriza el sistema para que pueda incorporar dichas varianzas, ajustando su output, y el usuario -o alguien previamente por él- selecciona los valores que le resultan más adecuados. Otra estrategia alternativa es crear sistemas independientes que implementen las varianzas de esas características. Es la estrategia que se sigue cuando se diseñan líneas de productos.

Por supuesto, la personalización conlleva trabajo, y los usuarios, por lo general, prefieren los sistemas que se ajustan automáticamente a sus preferencias o condiciones personales y ambientales. Veremos, sin embargo, que en ciertas edades los usuarios prefieren ejercer un control mayor y personal sobre sus herramientas.

En cualquier caso, los sistemas de información y documentación no son ajenos a toda esta lógica y, de hecho, la personalización es una de las líneas de investigación más importantes en este campo.

\subsection{Socialización}

Otra gran línea de desarrollo en los sistemas de información y documentación en estos tiempos es, sin duda, la incorporación de la computación social a las actividades y servicios de las bibliotecas, archivos y centros de documentación e información.

Esta tendencia se ha denominado Web 2.0 y despierta un gran interés entre la comunidad de los profesionales y científicos de la información, levantando incluso pasiones. Básicamente, consiste en desarrollar funcionalidades en los sitios web para que los usuarios puedan comunicarse y compartir información entorno a cuestiones de su interés obteniendo un feedback social, a partir de motivaciones sociales como la satisfacción por contribuir, la valoración mutua, la competencia, la exhibición de las propias cualidades, etc. Para ello, se utilizan tecnologías de comunicación cuyos resultados se presentan junto con la información de interés, y herramientas específicas para mostrar lo más popular, novedoso, impactante, etc.

Con ser importante y, aun siendo novedosa, la web social es una evolución lógica dentro del orden de las cosas. Desde una perspectiva antropológica, si se quiere a largo plazo, la llegada de la socialización al nuevo inter-medio que las nuevas tecnologías de la información y la comunicación han permitido crear —esto es, la Internet- es algo lógico y natural, y, en definitiva, una necesidad ontológica.

\section{La evolución psicosocial}

Efectivamente, el aspecto más interesante de la evolución humana durante la niñez y la adolescencia para un diseñador de sistemas de información es, probablemente, la amplitud e intensidad de los cambios que se producen en un relativamente corto periodo de tiempo.

Es, pues, una época privilegiada para estudiar la variabilidad humana. Además, por otra parte, y como fruto de los descubrimientos de las Psicología Evolutiva, se cuenta con un conocimiento profundo de las dimensiones de ese proceso de cambio, que se presentan en la tabla I.

Debe tenerse en cuenta, con todo, que las clasificaciones de las etapas del desarrollo cambian según las disciplinas -Pediatría, Psicología del Desarrollo, Política Educativa, etc.-, subdisciplinas -Psicología Cognitiva, Psicología de la Personalidad, etc.- y las diferentes escuelas y autores -Freud, Piaget, Erikson, etc. (v. g. Pa- 
palia et al., 2001). De manera análoga, las fronteras efectivas de cada fase pueden variar mucho dependiendo de los sujetos individuales. No obstante, esta clasificación permite dibujar las líneas fundamentales de desarrollo, que se repasan a continuación, como base para la pre- sentación de la tecnologías informáticas que pueden servir para abordarlas de cara a la personalización de los sistemas de información y bibliotecas digitales para niños y adolescentes.

\begin{tabular}{|c|c|c|c|c|c|}
\hline & Desarrollo físicc & & Cognitivo & \multirow{2}{*}{\multicolumn{2}{|c|}{$\begin{array}{l}\text { Social } \\
\text { Fuerte relación con los adultos } \\
\text { Creciente autonomía }\end{array}$}} \\
\hline $\begin{array}{l}\text { Primera infancia } \\
\text { I } b \\
18-36 \text { meses }\end{array}$ & \multirow{5}{*}{$\begin{array}{l}\text { Creciente } \\
\text { tamaño, } \\
\text { fuerza, control } \\
\text { motor, } \\
\text { equilibrio y } \\
\text { coordinación }\end{array}$} & $\begin{array}{l}\text { Habilidades } \\
\text { sensomotoras } \\
\text { iniciales }\end{array}$ & $\begin{array}{l}\text { Representación } \\
\text { perceptiva y conceptual } \\
\text { Adquisición del lenguaje }\end{array}$ & & \\
\hline $\begin{array}{l}\text { Primera infancia } \\
\text { II } \\
3 \text { a } 6 \text { años }\end{array}$ & & \multirow[t]{2}{*}{$\begin{array}{l}\text { Habilidades } \\
\text { motoras } \\
\text { mejoradas }\end{array}$} & $\begin{array}{l}\text { Imaginación } \\
\text { Conceptos 'crudos' } \\
\text { (clasificación a partir de } \\
\text { una sola característica) }\end{array}$ & \multicolumn{2}{|l|}{ Cooperación } \\
\hline $\begin{array}{l}\text { Segunda } \\
\text { infancia I } \\
6 \text { a } 9 \text { años }\end{array}$ & & & $\begin{array}{l}\text { Pensamiento lógico } \\
\text { Solución de problemas } \\
\text { concretos } \\
\text { Clasificación } \\
\text { multidimensional }\end{array}$ & \multicolumn{2}{|c|}{$\begin{array}{l}\text { Conformidad a reglas } \\
\text { Búsqueda de la competencia y } \\
\text { la autoestima }\end{array}$} \\
\hline $\begin{array}{l}\text { Segunda } \\
\text { infancia II }\end{array}$ & & $\begin{array}{l}\text { Fuerza } \\
\text { Estabilidad }\end{array}$ & Conceptos categoriales & \multirow{2}{*}{\multicolumn{2}{|c|}{$\begin{array}{l}\text { Creciente importancia del } \\
\text { grupo de iguales } \\
\text { Creciente apertura a } \\
\text { influencias sociales externas }\end{array}$}} \\
\hline $\begin{array}{l}\text { Infancia adulta } \\
9 \text { a } 12 \text { años }\end{array}$ & & Intranquilidad & Pensamiento histórico & & \\
\hline $\begin{array}{l}\text { Pre- } \\
\text { adolescencia } \\
12-14 \text { años }\end{array}$ & \multirow[t]{3}{*}{$\begin{array}{l}\text { Riesgos de } \\
\text { salud } \\
\text { Experiencias } \\
\text { emocionales } \\
\text { intensas }\end{array}$} & Pubertad & $\begin{array}{l}\text { Lógica formal } \\
\text { Lógica preposicional }\end{array}$ & \multirow{3}{*}{$\begin{array}{l}\text { Crítica } \\
\text { Búsqueda de } \\
\text { la identidad } \\
\text { Exploración } \\
\text { de roles } \\
\text { Creciente } \\
\text { conciencia y } \\
\text { compromiso } \\
\text { social }\end{array}$} & $\begin{array}{l}\text { Búsqueda de } \\
\text { afiliaciones y } \\
\text { liderazgo } \\
\text { Valores } \\
\text { abstractos }\end{array}$ \\
\hline $\begin{array}{l}\text { Adolescencia } \\
\text { 14-16 años }\end{array}$ & & $\begin{array}{l}\text { Despertar } \\
\text { sexual }\end{array}$ & Pensamiento crítico & & $\begin{array}{l}\text { Intensa vida } \\
\text { de grupo }\end{array}$ \\
\hline $\begin{array}{l}\text { Adolescencia } \\
\text { adulta } \\
16-19 \text { años }\end{array}$ & & $\begin{array}{l}\text { Emparejamiento } \\
\text { sexual }\end{array}$ & Pensamiento sistémico & & $\begin{array}{l}\text { Individuación } \\
\text { Preocupación } \\
\text { social y } \\
\text { vocacional }\end{array}$ \\
\hline
\end{tabular}

Tabla I. Evolución física y psicosocial en la niñez y la adolescencia.

Además del desarrollo físico —un crecimiento exponencial decreciente desde la concepción, sobre el que se sobrepone un cambio cualitativo en la pubertad, relacionado con la madurez reproductiva-, las tendencias principales de desarrollo psicológico de importancia fundamental para el desarrollo de sistemas de información y bibliotecas digitales para niños y adolescentes se pueden resumir en, fundamentalmente, dos: a) el lento y complejo camino hacia el pensamiento abstracto y crítico, que afecta de forma radical a la utilización de los vocabularios y lenguajes de búsqueda y, en general, a los aspectos simbólicos del interfaz de usuario y de las funcionalidades del sistema; y b) la consecución de la autonomía personal, dentro de los marcos permitidos por las constricciones culturales y, por ello, de forma progresiva al desarrollo de la responsabilidad y la implicación social, que comienza bajo la tutela estrecha de los padres y otras figuras parentales y se incrementa en en el marco de un creciente intercambio entre iguales.

En general, el proceso global puede caracterizarse como un camino de desarrollo físico y psicológico estructurado por una fuerte motivación de integración social, por más que el término 'social' deba entenderse en toda su complejidad y en todas sus contradicciones.

\subsection{El desarrollo cognitivo}

Las capacidades cognitivas varían enormemente a lo largo de todo el periodo, según mostraron Piaget y sus seguidores con gran detalle. Los dos polos del desarrollo son el pensamiento 
sensomotor y el abstracto, con un periodo intermedio denominado 'pensamiento concreto' que sienta el camino hacia mapas de la realidad más complejos y matizados (Piaget, 1950).

En el extremo inicial, los bebés prefieren contrastes fuertes, colores planos y distinciones muy claras entre fondo y forma. A esa edad, demasiada información o demasiado poca les desmotiva o aburre rápidamente. En general, cuanto más pequeños sean los niños, más cómodos y felices se sienten con interfaces simples.

Aunque la percepción y la cognición evolucionan de forma rápida e impresionante, éstas comienzan su desarrollo en un punto muy bajo, muy alejado de las competencias de un adulto normal. Esto no significa que los niños no puedan aprender cosas complicadas -lo hacen-, pero carecen de las competencias para conseguirlo eficientemente de forma autónoma y, por ello, necesitan supervisión adulta para hacerlo.

En el extremo final del periodo estudiado, los jóvenes adultos son capaces de apreciar temas y documentos muy difíciles, y conectarlos con las necesidades y valores sociales de forma abstracta y elaborada. De hecho, su capacidad abstractiva y deductiva se hipertrofia durante años, alejándoles hasta cierto punto de la realidad, a la que los sujetos estaban anclados sólidamente durante la etapa avanzada del pensamiento concreto.

\subsection{El desarrollo de la autonomía, el compromiso y la responsabilidad}

Como se adelantó en párrafos anteriores, la evolución social del niño desde la cuna a la vida adulta puede resumirse en dos tendencias complementarias: a) la autonomía, esto es, el desarrollo de las habilidades afectivas, cognitivas y sociales en un entorno seguro y afectivo que les permite alcanzar la autosuficiencia; y b) la socialización, esto es, la integración dentro de la sociedad a través de la familia y diversas clases de grupos.

En el punto de partida, los recién nacidos son totalmente dependientes y orientado a percepciones y objetos tangibles con relaciones y habilidades muy reducidas. En el punto de llegada, los adultos jóvenes son en general fuertes, desarrollados, capaces de pensamiento abstracto, socialmente y sexualmente interesados y capaces, y, por lo general, con muchas relaciones significativas.

A lo largo del itinerario, los sujetos en crecimiento se caracterizan - si todo va bien y, ciertamente, con conocidas crisis en el transcurso- por una insaciable curiosidad y una intensa actividad.

Por fin, durante este periodo de intenso crecimiento, los niños y adolescentes adquieren algunas características que les acompañarán durante toda su vida, entre las cuales es útil recordar la retroalimentación social, la competencia, la competición, la personalización, la afiliación, la identificación, el deseo de contribuir y la comunicación. Todas ellas, junto con la curiosidad y la actividad que se han señalado, son en sí mismas poderosas motivaciones que deben ser entendidas, servidas y usadas por la comunidad de desarrollo de bibliotecas digitales.

\section{Implicaciones para el diseño de sistemas de información y bibliotecas digitales}

Una vez establecidas las características psicosociales del desarrollo durante esta etapa, es posible conectarlas con algunos de los aspectos principales del diseño de bibliotecas infantiles y juveniles.

Cuatro factores al menos deberían considerarse: a) el contenido, que, obviamente, constituye el objeto de las necesidades, gustos y preferencias de los usuarios, y el foco de la actividad de una biblioteca, b) las propiedades motoras del interfaz, que aseguran que el usuario pueda manipular el sistema apropiadamente; c) las características preceptuales y simbólicas del interfaz y de los lenguajes de recuperación terminología y lenguajes de búsqueda- que deben adecuarse a las características específicas de la cognición infantil y juvenil, que afectan decisivamente a la usabilidad de las bibliotecas digitales y también en los procesos de capturar y mantener la atención; y d) las herramientas de "interconexión social".

Todos estos aspectos no deben contemplarse tan solo como instrumentos comunicacionales - sea entre lector y autor, persona y máquina o entre otros agentes-, sino también como instrumentos motivacionales: como herramientas que pueden promover e incrementar el uso del sistema y la satisfacción global del usuario. En particular, aseguran que los usuarios no se aburrirán o se desengancharan de un sistema que no entienden o que no son capaces de usar; que el sistema es capaz de ayudarles a encontrar los temas de su interés; y que proporciona acceso a retroalimentación y recompensas sociales. 


\subsection{El contenido}

Es muy difícil proporcionar en el breve marco de este artículo siquiera una panorámica esquemática de los intereses de un segmento tan diverso de usuarios. En realidad, los campos de atención de los niños y adolescentes son los mismos que los de los adultos - su mundo de referencia-, aunque, lógicamente, con las limitaciones de sus capacidades cognitivas y emocionales, $y$, por supuesto, de sus conocimientos.

Lo cierto es que la interacción de los niños con los documentos $-\mathrm{y}$, con ello, el potencial para que utilicen servicios bibliotecarios digitalespuede comenzar a una edad muy temprana, $y$, como se sabe, depende mucho de que los adultos les proporcionen la correcta motivación y guía. Los niños descubren objetos por ellos mismos, pero, principalmente, cuando juegan con adultos y otros niños. Los bebés desarrollan habilidades simbólicas muy tempranamente, y son capaces de identificar las imágenes como objetos alrededor de los doce meses; en unos pocos meses más son capaces de asignarles nombres.

Los temas de interés específicos cambian mucho a lo largo de todo el periodo. Los niños muy pequeños se interesan por los objetos, su significado y su localización, desaparición, recurrencia y movimiento (Bowerman, 2000). Miran a imágenes fijas, especialmente cuando alguien les cuenta una historia; $y$, como es bien conocido, son capturados muy pronto por los audiovisuales.

A lo largo del periodo, los niños crecen en su capacidad para procesar cada vez conocimientos más abstractos, especialmente cuando son vehiculados a través de historias y narraciones, como saben bien los padres y profesores.

Se muestran también muy activos e interesados en la búsqueda de información de su interés; y esta actividad se refuerza rápidamente si reciben información práctica de búsqueda en el marco de una educación activa y orientada a la solución de problemas.

En el futuro, la narración y las búsquedas orientadas a resolver necesidades específicas seguirán constituyendo el núcleo de su actividad de lectura como adultos, aunque de forma, lógicamente, más sofisticada.

\subsection{La interacción motora}

La interacción motora con sistemas multimedia es posible con un teclado simple y un ratón, y los niños y adolescentes disfrutan con ello, cada uno a su medida.
Los bebés y muestran muy pronto interés en interaccionar con ordenadores, y estas herramientas parecen especialmente útiles para su estadio de desarrollo cognitivo, pues a través del teclado y el ratón el procesamiento simbólico queda muy ligado a actividades motoras y al movimiento.

Como ocurre con la televisión, pueden pasar tiempos prolongados contemplando imágenes en movimiento y sonidos, probablemente porque esta experiencia es muy cercana a los procesos de descubrimiento espacial que ocurren en ambientes naturales, aunque de forma simplificada y con una gran concentración de información.

De esta manera, los ordenadores sirven de puente entre la actividades sensoriales y motoras en ambientes informacionales, aunque, por supuesto, estas herramientas deben utilizarse con cuidado y con contenidos apropiados, como en el caso de la televisión (Anderson and Evans, 2001).

\subsection{Las características perceptuales y} simbólicas de la interfaz

La evolución de la competencia perceptual y cognitiva tiene importantes implicaciones para el diseño de las interfaces y plantea un reto notable a las bibliotecas digitales que quieran proporcionar servicio a todo el periodo de la niñez y la adolescencia: para los niños más pequeños, las interfaces deben ser simples y con gran contraste -esto es, diferencia entre sus elementos-; para los adolescentes, deben tener en cuenta su necesidad de autoexpresar tanto su pertenencia social (afiliaciones) como su individualidad; y para los adultos jóvenes, el interfaz debería ser suficientemente flexible para soportar énfasis personalizados y actividades vocacionales - por ejemplo, mejorando la posibilidad de guardar búsquedas y perfiles-.

En general, un cierto grado de personalización será útil muy pronto, pues permite convertir la biblioteca digital en un espacio más personal, pero resulta más importante con niños adultos, adolescentes y, especialmente, adultos jóvenes.

Por otro lado, los aspectos gráficos y funcionales de la interfaz y de los lenguajes de búsqueda -con sus dos ramas, el vocabulario y las órdenes de búsqueda- no pueden ser separados, ya que forman una unidad de comunicación a través de diferentes canales y lenguajes complementarios.

El mejor ejemplo de esta realidad es el uso de vocabularios gráficos en los catálogos en línea para mostrar, precisar y sugerir la disponibilidad 
de materiales sobre los diferentes temas, una estrategia que en los entornos de búsqueda computacionales data al menos del proyecto Book House (Pejtersen, 1989) y que ha sido usado recientemente en proyectos tan notables como la International Children Digital Library (ICDL) (Drain, 2005).

Este acercamiento está justificado por la experiencia, pero también por resultados experimentales y modelos teóricos. Particularmente, algunos de los hallazgos sobre el desarrollo de conceptos pusieron en cuestión la capacidad de los niños para localizar la información con las clasificaciones y sistemas de materias y palabras clave normalmente usadas en los sistemas de información y las bibliotecas.

En concreto, Eleanor Rosch y sus colaboradores (1978) mostraron como los primeros conceptos se forman a través de procesos perceptivos básicos entorno a ejemplos centrales, a los que denominaron prototipos; y que dichos conceptos forman una capa de "conceptos naturales", a partir de la cual se produce posteriormente la construcción de conceptos más específicos y más generales mediante los mecanismos de la subordinación y supraordenación.

Así pues, los conceptos naturales pueden formarse fácilmente a partir de las características perceptuales de un buen ejemplo, pero tanto lo superordenados como los subordinados suelen requerir la enseñanza supervisada y el entrenamiento lógico y lingüístico. Por ejemplo, los niños aprenden rápidamente el concepto de persona, que rápidamente son capaces de nombrar con conceptos naturales como mamá, papá, tío, etc.; después comienzan a discriminar mediante nombres propios, y empiezan a formar categorías fácilmente identificables, como los bomberos con sus camiones y trajes rojos.

Con el tiempo, comienzan a descubrir grupos naturales como las familias, pero solo lentamente $-y$ muchas veces con dificultad- aprende las categorías supraordenadas más abstractas como sociedad, clase, etc.

Toda esta perspectiva sobre la formación de conceptos cuestiona, por ejemplo, el uso de clasificaciones jerárquicas con niños pequeños para facilitar la navegación conceptual temática, y sugiere que el nivel de entrada a una clasificación debe ser siempre el nivel de los conceptos naturales y, posteriormente, el nivel de abstracción adecuado para su edad.

Desde otro punto de vista, el modelo desarrollado por Piaget (1950) proporciona también un fuerte apoyo teórico a la utilización de lenguajes de búsqueda gráficos en la primera infancia y concretos en la segunda, así como para la progresiva introducción de nuevas funcionalidades de forma adecuada a la edad del usuario.

La necesidad de incorporar sistemas de ayuda contextuales extensos es apoyada también por las investigaciones de la escuela de la psicología genética, conectadas con el desarrollo del conocimiento experto en dominios específicos. Básicamente, la evidencia muestra que las capacidades lógicas avanzadas solo se emplean con confianza en ámbitos en los que se ha alcanzado un conocimiento experto.

Puesto que los niños tienen un conocimiento más limitado del mundo y capacidades operacionales más limitadas, necesitan de más apoyo contextual al buscar en la biblioteca y utilizarla. No es solo cuestión de simplificar -lo que conduce muchas veces a una ejecución empobrecida-, sino, sobre todo, de proporcionar información contextual y múltiples caminos alternativos para alcanzar los resultados, pues normalmente son capaces de sobreponerse a sus carencias en entornos flexibles que les permitan desarrollar su intensa actividad.

\subsection{El problema de la interacción social y las herramientas "sociales"}

Las necesidades sociales varían mucho a lo largo de un periodo de desarrollo tan amplio y complejo, como se pudo apreciar en la tabla I. Sin embargo, el argumento principal permanece constante. Aunque puedan parecer tendencias contrapuestas, la identidad y la pertenencia, la afirmación individual y la responsabilidad, son características que se desarrollan conjuntamente.

Hacia los cinco o seis años, aparece una endencia que acompañará a los niños gran parte de su vida: los niños disfrutan mostrando su competencia en el desempeño de sus habilidades y compitiendo entre ellos. Muy pronto, preferirán competir en grupos, y se mostrarán cada vez más preocupados por compararse ellos y sus gustos con los de sus iguales. Algunos años más tarde, al final de la niñez, se mostrarán más interesados en la vida social más amplia y en la pertenencia social, transcendiendo las fronteras de sus familias y comunidades locales. En la adolescencia, esos grupos necesitan más independencia del mundo de los adultos, que les permita desarrollar sus propias culturas. Siguen estando muy interesados -incluso más- en el mundo de los adultos, aunque tomando distancia y asumiendo una postura muy crítica. Al final del periodo, se sumergen en decisiones vocacionales - profesionales, familiares y persona- 
les- que suponen y requieren un intenso compromiso con la sociedad adulta.

De nuevo, es necesario señalar que estas características acompañarán al adulto durante toda su vida, aunque integradas en un creciente sentido de la individualidad y la afiliación: retroalimentación social, personalización-identificación, contribución, comunicación...

Pues bien, es posible identificar diversas tecnologías susceptibles de apoyar cada uno de estos aspectos de la interacción social y que pueden ser aplicados $-\mathrm{y}$ de hecho lo están siendo- a las bibliotecas digitales infantiles y juveniles.

Para favorecer la retroalimentación social, existe un conjunto de conceptos tecnológicos orientados tanto a la retroalimentación por pares como desde una posición de autoridad. En el primer grupo, el de la retroalimentación entre iguales, son muy utilizadas las votaciones, las reseñas, los ítems recuperados conjuntamente anteriormente, las listas de documentos más leídos (más populares) o más leídos recientemente... En el campo de la retroalimentación desde una posición de autoridad, se utilizan también las noticias, las recomendaciones, las reseñas, y otros instrumentos semejantes.

En el campo de la personalización y de la identidad social, se proporcionan herramientas tanto orientadas a sujetos como a grupos. La personalización, como se vio al principio, consiste en la posibilidad que ofrece un sistema de modificar el interfaz de usuario para acomodarlo a preferencias individuales en el uso de colores, disposición y presencia de elementos, ontologías de búsqueda, etc., y también en la oferta de tecnologías push como los boletines o el RSS. Más raros, aunque igualmente interesantes desde el punto de vista que hemos examinado, son los instrumentos de "personalización grupal", orientados a acomodar aspectos idiosincrásicos de la identidad de grupo, clase, cultural o étnica de los usuarios.

La necesidad de contribuir al trabajo común se puede facilitar de dos maneras: a) proporcionando la posibilidad real de ofrecer las propias ideas mediante la publicación de nuevos recursos, opiniones, reseñas, etc.; y b) ofreciendo la oportunidad de crear en grupo mediante tecnologías de trabajo en grupo como los wikis, etc.

Por fin, la necesidad de comunicarse - tanto entre iguales como dentro de una jerarquía de autoridad o supervisión - se facilita mediante tecnologías como el chat, la videoconferencia y sus combinaciones. Como es sabido, estas tecnologías requieren de supervisión y control adulto adecuado a la edad de los participantes.

De hecho, la utilización de las tecnologías que se han revisado constituyen la explicación del éxito y la popularidad de los portales de interacción social, como eBay, Amazon o YouTube.

Las bibliotecas digitales especializadas en niños y adolescentes llevan ventaja en la aplicación de estas tecnologías, porque normalmente reconocen entre sus fines el objetivo explícito de favorecer las competencias comunicaciones e informacionales entre sus jóvenes usuarios, mientas que las bibliotecas públicas y especializadas suelen enfatizar más aspectos relacionados con la idoneidad de sus colecciones de documentos. De hecho, las bibliotecas educativas necesitan desarrollar actividades para promover la creación y participación de los usuarios para alcanzar sus objetivos. Por ello, muchas bibliotecas digitales para niños y adolescentes proclaman y practican de forma abierta su carácter "social". Así, por ejemplo, Chilias incluye talleres de cuenta cuentos y concursos (Lorente, 2005).

La socialización es una poderosa motivación en sí misma que puede ayudar a comunicar contenidos difíciles $y$, en principio, poco atractivos para una determinada audiencia. En este sentido, refieriéndose a las bibliotecas digitales de matemáticas, Chen y Doty (2005) resumen la importancia de favorecer la interacción social dentro del programa de las bibliotecas digitales infantiles y juveniles:

The communicative power of computers and digital libraries is what offers the best hope for enhancing mathematics education, not simple comprehensive collections and complex retrieval algorithms.

\section{Conclusiones}

Las evoluciones recientes en el campo de los sistemas de información y las bibliotecas digitales no surgen de la nada, sino que son la respuesta moderna a necesidades humanas de siempre, aprovechando las oportunidades que ofrecen las nuevas tecnologías de la información y la comunicación.

El estudio de las necesidades que subyacen a los procesos de información es necesario para anclar los avances prácticos de las tecnologías bibliotecarias digitales dentro de una comprensión integral del ser humano y de la vida social. Solo así seremos capaces de integrar los aspectos tecnológicos y prácticos del desarrollo de los sistemas de información y las bibliotecas digitales con un abordaje propiamente científico del mundo de la información, que no se puede 
explicar fuera del marco antropológico en el que evoluciona.

No es extraño que los dos principales motores de la evolución psicológica humana —la individuación y la socialización- estén precisamente en la raíz de las dos tendencias tecnológicas más importantes en los sistemas de información y las biblitecas digitales: la personalización y la individualización. De lo que se puede concluir que el conocimiento de las aportaciones de las ciencias humanas y las humanidades sobre estos temas deberían ser tenidos en cuenta por los expertos en el diseño y el uso de sistemas de información y bibliotecas digitales.

\section{Referencias}

Anderson, D. R.; M. K., Evans (2001). Peril and Potential of Media for Infants and Toddlers. // Zero to Three. 22:2 (2001) 10-16.

Bowerman, M. (2000). Where do children's word meanings come from? Rethinking the role of cognition in early semantic development. // Nucci, L. P.; Saxe, G.; Turiel, E. (Eds.). Culture, thought, and development. Mahwah, $\mathrm{NJ}$ : Erlbaum, 2000. 199-230.

Centro de Investigación y Documentación Educativa (2002). Los hábitos lectores de los adolescentes españoles. Boletín CIDE de temas educativos, 10. Monográfico.

Centro Internacional del Libro Infantil y Juvenil (2005). ¿Nuevas lecturas? ¿nuevas formas de leer?: lectura y escritura multimedia en las bibliotecas públicas y escolares. Madrid: Fundación Germán Sánchez Ruipérez, 2005.

Chen, H.,; Doty, P. (2005). A conceptual framework for digital libraries for k-12 mathematics education: part 1 , information organization, information literacy, and integrated learning. // The Library Quarterly. 75:3 (2005) 231-161.

Drain, A. (2005). What children can teach us: developing digital libraries for children with children. // Library Quarterly. 75:1 (2005) 20-41.

García Marco, Francisco Javier (2008). Lo nuevo y lo de siempre en las bibliotecas digitales: reflexiones desde el análisis de los servicios para niños y adolescentes. // Scire. 14:2 (jul.-dic. 2008).

Lorente, M. (2005). Desarrollo y aplicación del proyecto CHILIAS en la Diputación de Barcelona. // Centro Internacional del Libro Infantil y Juvenil (2005). ¿Nuevas lecturas? ¿nuevas formas de leer?; lectura y escritura multimedia en las bibliotecas públicas y escolares. Madrid: Fundación Germán Sánchez Ruipérez, 2005. 35-50.

Wendkos Olds, R.; Feldman, R. D. (2004). A child's world; infancy through adolescente (Updated 9th ed.). New York: McGraw-Hill, 2004.

Pejtersen, A. M. (1989). The Book house; modelling users' needs and search strategies as a basis for system design. Roskilde: Risø National Laboratory, 1989.

Piaget, J. (1950). Introduction à l'épistémologie génétique. 3 Vols. Paris: Presses Universitaires de France, 1950.

Rosch, E. ; Lloyd, B. B. (eds.). Cognition and categorization. Hillsdale, N.J.: Lawrence Erlbaum, 1978.

Toffler, Alvin (1980). The Third wave. 1st ed. New York: Morrow, 1980, 1980. 544 p. 